Proc Indian Natn Sci Acad $\mathbf{8 1}$ No. 1 February 2015 Special Issue, pp. 233-236

(C) Printed in India.

DOI: $10.16943 /$ ptinsa/2015/v81i1/48075

\title{
Characterization of Polyethylene Terephthalate (PET) Detector to Search for Rare Events in Cosmic Rays

\author{
S DEY ${ }^{1}$ A MAULIK ${ }^{1,2, *}$, SIBAJI RAHA ${ }^{1,2}$, SWAPAN SAHA ${ }^{1,2}$ and D SYAM ${ }^{1,3}$ \\ ${ }^{1}$ Center for Astroparticle Physics and Space Science, Bose Institute, Kolkata 700 091, India \\ ${ }^{2}$ Department of Physics, Bose Institute, Kolkata 700 009, India \\ ${ }^{3}$ Department of Physics, Barasat Government College, Kolkata 700 124, India
}

(Received on 07 May 2014; Accepted on 05 August 2014)

\begin{abstract}
A particular brand of commercially available plastic, identified as polyethylene terephthalate (PET) has been used as a Nuclear Track Detector (NTD) to detect heavy charged particles. It was found that PET has a much higher detection threshold compared to other commercially available NTDs, making PET particularly suitable for detecting rare events in cosmic rays. To characterize and calibrate PET, systemetic studies were carried out using ions from various accelerators in India and Europe. Results of those studies have shown that PET can be effectively used as a charge particle detector with good energy and charge resolution.
\end{abstract}

\section{Key Words : PET; NTD; SQM; Strangelet}

\section{Introduction}

According to many theorists, Strange Quark Matter (SQM) containing almost equal numbers of up, down and strange quarks constitutes the true ground state of quantum chromodynamics (Witten, 1984). SQM, if stable, is almost unavoidable in the core of dense stellar objects like neutron stars and small lumps of SQM called strangelets may be produced when such objects in a binary system collide. Such strangelets will then be accelerated by usual cosmic ray acceleration mechanisms. Theoretical studies have pointed to a significant measurable strangelet flux in our part of the galaxy. So an ideal place to look for such exotic matter is in cosmic rays. An unique experimental signature for strangelets is a highly unusual charge to mass ratio $(\mathrm{Z} / \mathrm{A} \ll 1 / 2)$. According to one model of strangelet propagation (Banerjee et al., 2000) through the earths atmosphere, an initially small strangelet will grow in size by preferentially absorbing neutrons

*Author for Correspondence : E-mail: atanu@iopb.res.in 
over protons by interacting with the nuclei of atmospheric atoms. At the same time they lose energy by ionization of the surrounding media but should be detectable at high mountain altitudes with a very low flux.

To look for such rare events at very high mountain altitudes Nuclear Track Detectors (NTDs) are ideal. NTDs are inexpensive, simple to use, they do not require power for their operation and have natural thresholds of detection. NTDs are dialectric solids. Ionizations and excitations caused by charged particles on their passage through such materials leave behind narrow damage trails. On being treated by a suitable chemical reagent (enchant) material along the damage trails are etched out at a much faster rate $\mathrm{V}_{T}$ called track etch rate compared to the rate of etching $\mathrm{V}_{B}$ called bulk etch rate of the undamaged bulk material. The etch pits so formed can be closely approximated by a geometrical cone with the damage trail as its axis and is observable under an optical microscope. By studying the geometry of etch pits the charge of the ion forming the track can be determined. The range of the particle inside the detector gives energy. For our work we have chosen a particular brand of polymer, called Polyethylene Terephthalate (PET) as NTD. It is found to have a much higher detection threshold compared to many other commercially available polymer detectors like CR-39, Lexan etc. and as such is particularly suitable for rare event search in cosmic rays by eliminating the huge low $\mathrm{Z}$ background. A detector needs to be calibrated using known ions before being used to detect any unknown particle. So we have characterized and calibrated PET by exposing PET films to ions from different particle accelerators.

\section{Experiment}

To calibrate PET, detector films were exposed to $3.4 \mathrm{MeV} / \mathrm{A}{ }^{16} \mathrm{O}, 2.7 \mathrm{MeV} / \mathrm{A},{ }^{56} \mathrm{Fe}$ and $3.9 \mathrm{MeV} / \mathrm{A}{ }^{32} \mathrm{~S}$ beams from the Pelletron accelerator at IUAC, New Delhi, $11.1 \mathrm{MeV} / \mathrm{A}{ }^{238} \mathrm{U}$ ions from GSI, Germany and 2.82 MeV/A ${ }^{129} \mathrm{Xe}$ and ${ }^{78} \mathrm{Kr}$ ions from REX-ISOLDE, CERN, Switzerland. The ${ }^{78} \mathrm{Kr}$ beam contained an admixture of ${ }^{49} \mathrm{Ti}$ ions which provided an additional data point for calibration. All exposed samples were etched in $6.25 \mathrm{~N} \mathrm{NaOH}$ solution kept at $55^{\circ} \mathrm{C}$ which was found to the be ideal etching condition for PET (Bhowmik et al., 2011).

\section{Results and Discussions}

Fig. 1 shows track images due to ${ }^{78} \mathrm{Kr}$ as well as ${ }^{49} \mathrm{Ti}$ on PET after $2 \mathrm{hr}$ etching. Different track diameters corresponding to the two ions are evident from Fig. 1(A) with the microscope focussed on the detector surface. Fig. 2(B) shows that the end of track is reached for ${ }^{49} \mathrm{Ti}$ ions but not for ${ }^{78} \mathrm{Kr}$ with the microscope focussed at a depth of $8.2 \mu \mathrm{m}$. This implies different track lengths for the two ions (Dey et al., 2011). 

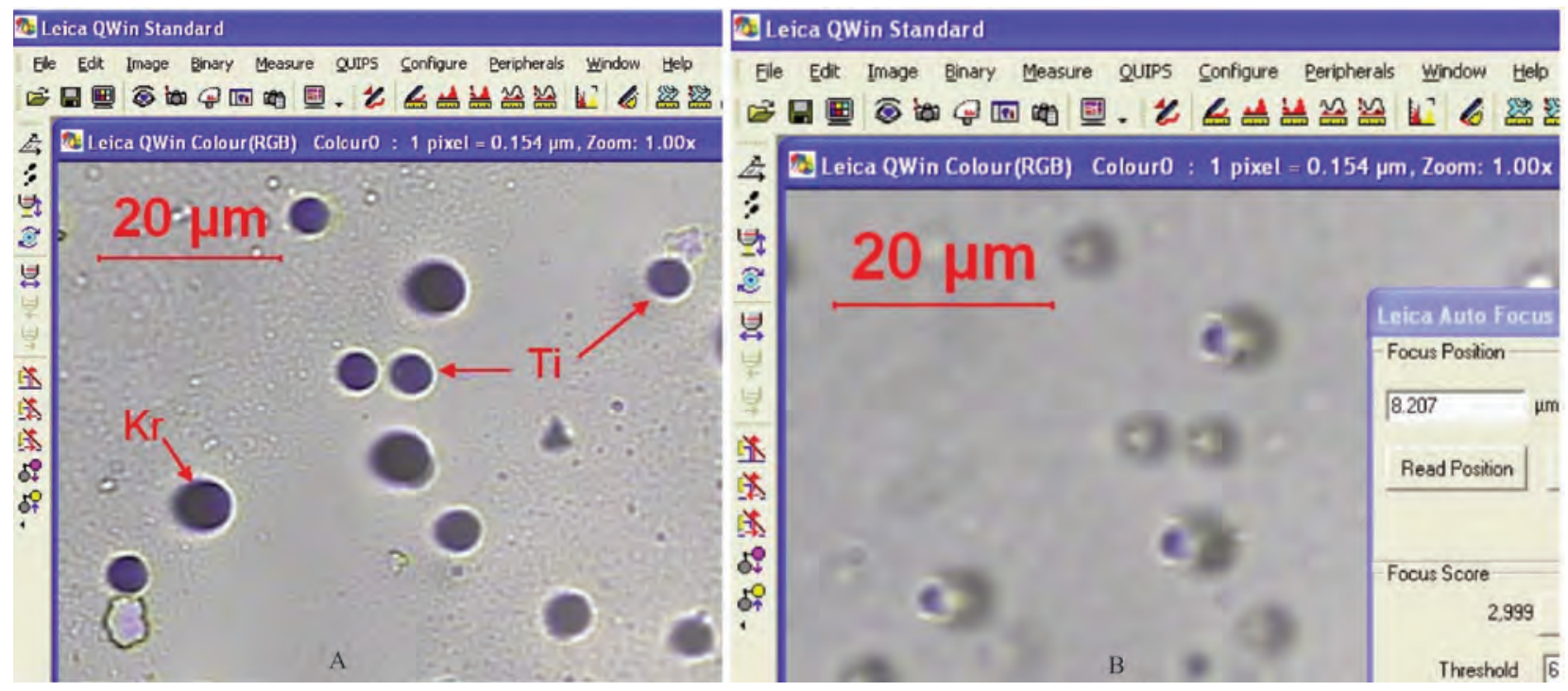

Fig. 1: The different track dimensions for ${ }^{78} \mathrm{Kr}$ and ${ }^{49} \mathrm{Ti}$ can be seen with the microscope focussed (A) on the surface and (B) at a depth of $8.2 \mu \mathrm{m}$.

By combining the $V_{T} / V_{B}$ values for ${ }^{129} \mathrm{Xe},{ }^{78} \mathrm{Kr},{ }^{49} \mathrm{Ti}$ as well as those for ${ }^{16} \mathrm{O},{ }^{32} \mathrm{~S},{ }^{56} \mathrm{Fe},{ }^{238} \mathrm{U}$ ions, we can get a calibration curve for PET as shown in Fig. 2. The specific energy losses $d E / d x$ of the incident ions at different energies were obtained using SRIM (SRIM, Version 2003.06).

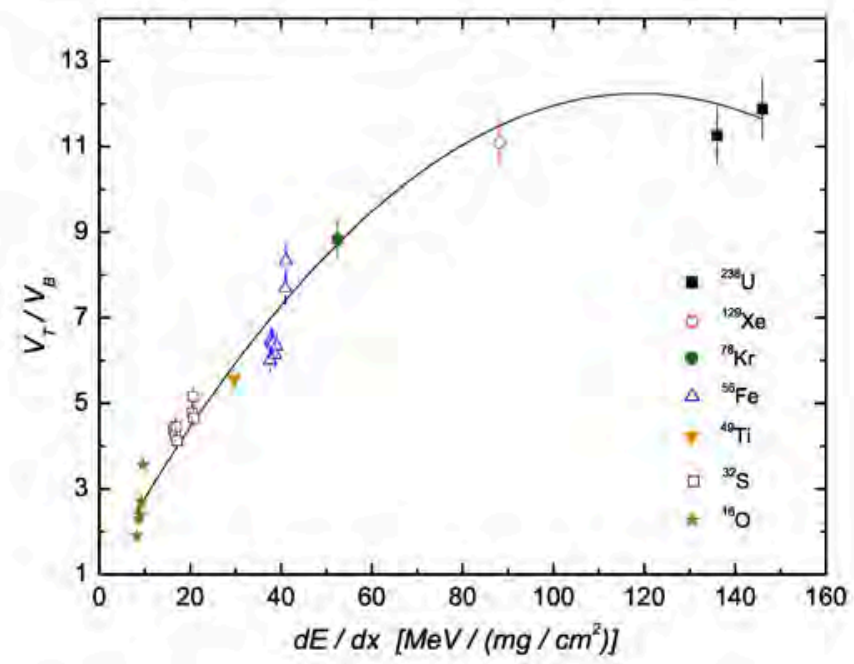

Fig. 2: Calibration curve for PET

Our work clearly demonstrates that PET can be effectively used as a charged particle detector with good energy and charge resolution. Also crucially, PET has a very high detection threshold and can be particularly 
useful in cases where there is a requirement for detecting high $\mathrm{Z}$ particles against a low $\mathrm{Z}$ background. Another advantage for PET is its significantly low cost compared to other commercially available NTDs. Cost considerations become particularly important if one intends to setup large area passive detector arrays which we plan to do in future.

\section{Acknowledgement}

The work is funded by IRHPA (Intensification of Research in High Priority Areas) Project (IR/S2/PF01/2011 dated 26.06.2012) of the Science and Engineering Research Council (SERC), DST, Government of India, New Delhi.

\section{References}

1. Banerjee S, Ghosh SK, Raha S and Syam D (2000) Can cosmic strangelets reach the earth?, Phys Rev Lett 85 1384

2. Bhowmik D, Dey S, Maulik A, Raha S, Saha S, Saha SK, Syam D (2011) Characterization and calibration of a SSNTD for heavy-ion detection and strangelet search in cosmic rays, Nuclear Instrum and Meth B 269197

3. Dey S, Gupta D, Maulik A, Raha S, Saha SK, Syam D, Pakarinen J, Voulot D and Wenander F (2011) Calibration of a solid state nuclear track detector (SSNTD) with high detection threshold to search for rare events in cosmic rays, Astroparticle Physics 34805

4. Witten E (1984) Cosmic separation of phases, Phys Rev D30 272

5. Ziegler JF and Biersack JP (Version: 2003.26) The Stopping and Range of Ions in Matter (SRIM Computer Code). 